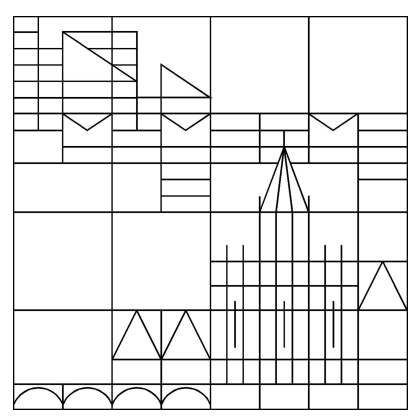

\title{
Nonlinear stability of Ekman boundary layers
}

\author{
Matthias Hess \\ Matthias Hieber \\ Alex Mahalov \\ Jürgen Saal
}

Konstanzer Schriften in Mathematik und Informatik

Nr. 242, Februar 2008

ISSN $1430-3558$

Konstanzer Online-Publikations-System (KOPS)

URL: http://www.ub.uni-konstanz.de/kops/volltexte/2008/4860/

URN: http://nbn-resolving.de/urn:nbn:de:bsz:352-opus-48606

\author{
(C) Fachbereich Mathematik und Statistik \\ (C) Fachbereich Informatik und Informationswissenschaft \\ Universität Konstanz \\ Fach D 188, 78457 Konstanz, Germany \\ E-Mail: preprints@informatik.uni-konstanz.de \\ WWW: http://www.informatik.uni-konstanz.de/Schriften/
}





\title{
NONLINEAR STABILITY OF EKMAN BOUNDARY LAYERS
}

\author{
MATTHIAS HESS, MATTHIAS HIEBER, ALEX MAHALOV, JÜRGEN SAAL
}

\begin{abstract}
Consider the initial value problem for the three dimensional Navier-Stokes equations with rotation in the half-space $\mathbb{R}_{+}^{3}$ subject to Dirichlet boundary conditions as well as the Ekman spiral which is a stationary solution to the above equations. It is proved that the Ekman spiral is nonlinearly stable with respect to $L^{2}$-perturbations provided the corresponding Reynolds number is small enough. Moreover, the decay rate can be computed in terms of the decay of the corresponding linear problem.
\end{abstract}

\section{INTRODUCTION}

Consider the the initial value problem for the three dimensional Navier-Stokes equations with rotation in the half-space $\mathbb{R}_{+}^{3}$ subject to Dirichlet boundary conditions, i.e. the set of equations

$$
\left\{\begin{array}{rlrl}
\partial_{t} u-\nu \Delta u+\Omega \mathbf{e}_{3} \times u+(u \cdot \nabla) u+\nabla p & =0, & & t>0, x \in \mathbb{R}_{+}^{3}, \\
\operatorname{div} u & =0, & & t>0, x \in \mathbb{R}_{+}^{3}, \\
u\left(t, x_{1}, x_{2}, 0\right) & =0, & t>0, x_{1}, x_{2} \in \mathbb{R}, \\
u(0, x) & =u_{0}, & & x \in \mathbb{R}_{+}^{3},
\end{array}\right.
$$

where $u=\left(u^{1}, u^{2}, u^{3}\right)$ denotes the velocity field and $p$ the pressure of an incompressible, viscous fluid. Here, $\mathbf{e}_{3}$ denotes the unit vector in $x_{3}$-direction, $\nu>0$ the viscosity of the fluid, and the constant $\Omega \in \mathbb{R}$ is called the Coriolis parameter which is equal to twice the frequency of rotation around the $x_{3}$ axis.

It is well known that the above system has a stationary solution which can be expressed even explicitly as

$$
\begin{aligned}
& u_{E}\left(x_{3}\right)=u_{\infty}\left(1-e^{-x_{3} / \delta} \cos \left(x_{3} / \delta\right), e^{-x_{3} / \delta} \sin \left(x_{3} / \delta\right), 0\right)^{T}, \\
& p_{E}\left(x_{2}\right)=-\Omega u_{\infty} x_{2},
\end{aligned}
$$

where $\delta$ is defined by $\delta:=\left(\frac{2 \nu}{\Omega}\right)^{1 / 2}$ and $u_{\infty} \geq 0$ is a constant. This stationary solution of equation (1.1) is called in honour of the swedish oceanograph V.W. Ekman, the Ekman spiral; see [8]. It describes mathematically rotating boundary layers in geophysical fluid dynamics (atmospheric and oceanic boundary layers) between a geostrophic flow and a solid boundary at which the no slip boundary condition applies. Moreover, $\delta$ denotes the thickness of the layer. In the geostrophic flow region corresponding to large $x_{3}$, there is a uniform flow with velocity $u_{\infty}$ in the $x_{1}$ direction. Associated with $u_{\infty}$, there is a pressure gradient in the $x_{2}$-direction. The Ekman spiral in $\mathbb{R}_{+}^{3}$ matches this uniform velocity for large $x_{3}$ with the no slip boundary condition at $x_{3}=0$, i.e. we have $u_{E}(0)=0$ and

$$
u_{E}\left(x_{3}\right) \rightarrow\left(u_{\infty}, 0,0\right) \quad \text { provided } \quad x_{3} \rightarrow \infty .
$$

In this paper we are interested in stability questions for the Ekman spiral. More precisely, we consider perturbations of the Ekman spiral by functions $u$ solving the above equation (1.1). To this end, set

$$
w:=u-u_{E}, \quad \text { and } \quad q:=p-p_{E} .
$$

2000 Mathematics Subject Classification. 35,76D05,76E07.

Key words and phrases. Ekman spiral, Navier-Stokes, nonlinear stability. 
Since $\left(u_{E}, p_{E}\right)$ is a stationary solution of $(1.1)$, the pair $(w, q)$ formally satisfies the equations (1.4)

$$
\left\{\begin{aligned}
\partial_{t} w-\nu \Delta w+\Omega \mathbf{e}_{3} \times w+\left(u_{E} \cdot \nabla\right) w+w_{3} \partial_{3} u_{E}+(w \cdot \nabla) w+\nabla q & =0, \quad t>0, x \in \mathbb{R}_{+}^{3}, \\
\operatorname{div} w & =0, \quad t>0, x \in \mathbb{R}_{+}^{3}, \\
w\left(x_{1}, x_{2}, 0\right) & =0, \quad t>0, x_{1}, x_{2} \in \mathbb{R}, \\
w(0, x) & =w_{0}, \quad x \in \mathbb{R}_{+}^{3},
\end{aligned}\right.
$$

where $w_{0}=u_{0}-u_{E}$.

It is natural to conjecture that there exists a critical Reynolds number $R e_{c}$ with the property that if $R e<R e_{c}$, then the perturbed nonlinear flow is stable and that the flow is unstable provided $R e>$ $R e_{c}$. Here $R e=u_{\infty} \delta \nu^{-1}$ denotes the Reynolds number of the given fluid. It seems that there is no mathematical proof of this statement so far.

Considering the linearized version of our problem, i.e.

$$
\left\{\begin{aligned}
\partial_{t} w-\nu \Delta w+\Omega \mathbf{e}_{3} \times w+\left(u_{E} \cdot \nabla\right) w+w^{3} \partial_{3} u_{E}+\nabla q & =0, \quad t>0, x \in \mathbb{R}_{+}^{3}, \\
\operatorname{div} w & =0, \quad t>0, x \in \mathbb{R}_{+}^{3}, \\
w\left(x_{1}, x_{2}, 0\right) & =0, \quad t>0, x_{1}, x_{2} \in \mathbb{R}, \\
w(0, x) & =w_{0}, \quad x \in \mathbb{R}_{+}^{3}
\end{aligned}\right.
$$

we remark that linear stability results for the Ekman spiral can be obtained fairly easily by energy methods, again of course for small Reynolds number. Results on linear instability of $w$ for large Reynolds number are more difficult to obtain. It was shown in [7] that in the case of flows between infinite layers, there exists a sequence of approximate solutions to (1.4) which is nonlinearly unstable for sufficiently large Reynolds numbers in the sense of [7].

Recently it was shown in [12] that the nonlinear equation (1.4) admits a unique, local mild solution for all non decaying initial data belonging to a certain Besov space. In this paper, we consider the problem of global weak solutions to (1.4) and study also their nonlinear stability behaviour for initial data belonging to $L_{\sigma}^{2}\left(\mathbb{R}_{+}^{3}\right)$.

We show in our first main result that there exists a global weak solution to the above set (1.4) of nonlinear equations provided the Reynolds number $R e=u_{\infty} \delta \nu^{-1}$ is small enough. Secondly, assuming this condition, for every initial data $w_{0} \in L_{\sigma}^{p}\left(\mathbb{R}_{+}^{3}\right)$, there exists at least one global weak solution $w$ to (1.4) such that

$$
\lim _{t \rightarrow \infty}\|w(t)\|_{2}=0
$$

which shows in particular that the Ekman spiral is nonlinearly stable with respect to $L^{2}$-perturbations. Moreover, it is even possible to estimate the decay rate. Indeed, roughly speaking, if $R e$ is small enough, $0<\alpha \leq \frac{1}{4}$ and $w_{0} \in L_{\sigma}^{2}\left(\mathbb{R}_{+}^{3}\right)$ satisfies

$$
\left\|e^{-t A_{S C E}} w_{0}\right\|_{2}=O\left(t^{-\alpha}\right)
$$

then there exists at least one global weak solution $w$ to the nonlinear problem (1.1) having the same decay rate. A similar result applies for arbitrary $\alpha>0$. Here $e^{-t A_{S C E}}$ denotes the semigroup on $L_{\sigma}^{2}\left(\mathbb{R}_{+}^{3}\right)$ generated by the Stokes-Coriolis-Ekman operator in $L_{\sigma}^{2}\left(\mathbb{R}_{+}^{3}\right)$ defined in the following section.

Our approach is inspired by the methods developed by Miyakawa and Sohr [15] and Borchers and Miyakawa [3],[2] in order to construct weak solutions to the Navier-Stokes equations - without rotational effects - on exterior domains. For more information on the Navier-Stokes equations with rotational effect, we refer to [13] or [1] and [4]. Although the assertions of our two main results are stated completely within the $L^{2}$-framework, our proof needs so-called maximal $L^{p}$-regularity estimates for the StokesCoriolis-Ekman operator in the halfspace $\mathbb{R}_{+}^{3}$ for $p \neq 2$. We sketch the proof of these estimates in Section 3. 


\section{Preliminaries and Main Results}

For $1<p<\infty$ denote by $P$ the Helmholtz projection from $L^{p}\left(\mathbb{R}_{+}^{3}\right)$ to $L_{\sigma}^{p}\left(\mathbb{R}_{+}^{3}\right)$. We then may rewrite equation (1.4) as an evolution equation in $L_{\sigma}^{p}\left(\mathbb{R}_{+}^{3}\right)$ of the form

$$
\left\{\begin{aligned}
w^{\prime}+A_{S C E} w+P(w \cdot \nabla) w & =0, \quad t>0 \\
w(0) & =w_{0},
\end{aligned}\right.
$$

where the Stokes-Coriolis-Ekman operator $A_{S C E}$ in $L_{\sigma}^{p}\left(\mathbb{R}_{+}^{3}\right)$ is defined by

$$
\left\{\begin{aligned}
A_{S C E} w & :=P\left(-\nu \Delta w+\Omega \mathbf{e}_{3} \times w+\left[\left(u_{E} \cdot \nabla\right) w+w_{3} \partial_{3} u_{E}\right]\right)=\left(A_{S}+A_{C}+A_{E}\right) w \\
D\left(A_{S C E}\right) & :=W^{2, p}\left(\mathbb{R}_{+}^{3}\right) \cap W_{0}^{1, p}\left(\mathbb{R}_{+}^{3}\right) \cap L_{\sigma}^{p}\left(\mathbb{R}_{+}^{3}\right) .
\end{aligned}\right.
$$

It follows for example from the results in [6] that the usual Stokes operator $A_{S, p}=-P \Delta$ generates a bounded analytic semigroup $e^{-t A_{S, p}}$ on $L_{\sigma}^{p}\left(\mathbb{R}_{+}^{3}\right)$ for all $p \in(1, \infty)$. By standard perturbation theory, we see that the Stokes-Coriolis-Ekman operator generates also an analytic semigroup $e^{-t A_{S C E, p}}$ on $L_{\sigma}^{p}\left(\mathbb{R}_{+}^{3}\right)$. In particular, after possible rescaling, the square root $\left(A_{S C E, 2}\right)^{1 / 2}$ of $A_{S C E, 2}$ is a well defined operator in $L^{2}\left(\mathbb{R}_{+}^{3}\right)$. This allows us to define a weak solution to (1.4) as follows. For simplicity of notation, we omit the index 2 and write in the following $A_{S C E}=A_{S C E, 2}$.

Definition 2.1. Let $w_{0} \in L_{\sigma}^{2}\left(\mathbb{R}^{3}\right)$ and $f \in L^{2}\left((0, T) ; L_{\sigma}^{2}\left(\mathbb{R}_{+}^{3}\right)\right.$ for all $T>0$. We call $w:[0, \infty) \rightarrow L_{\sigma}^{2}\left(\mathbb{R}_{+}^{3}\right)$ a weak solution of equation (1.4) if for all $T>0$,

i) $w \in L^{\infty}\left((0, T) ; L_{\sigma}^{2}\left(\mathbb{R}_{+}^{3}\right)\right) \cap L^{2}\left((0, T) ; D\left(A_{S C E}^{1 / 2}\right)\right)$ and

ii)

$$
\begin{aligned}
& -\int_{0}^{T}\langle w, \phi\rangle h^{\prime}(t) d t+\nu \int_{0}^{T}\langle\nabla w, \nabla \phi\rangle h(t) d t+\int_{0}^{T}\left\langle\left(u_{E} \cdot \nabla\right) w, \phi\right\rangle h(t) d t \\
+ & \int_{0}^{T}\left\langle w_{3} \cdot \partial_{3} u_{E}, \phi\right\rangle h(t) d t+\Omega \int_{0}^{T}\left\langle\mathbf{e}_{3} \times w, \phi\right\rangle h(t) d t+\langle w \cdot \nabla w, \phi\rangle h(t) d t=\left\langle w_{0}, \phi\right\rangle h(0),
\end{aligned}
$$

holds for all $\phi \in D\left(A_{S C E}^{1 / 2}\right)$ and all $h \in C^{1}([0, T], \mathbb{R})$ with $h(T)=0$.

Note that the sixth term on the left hand side above is meaningful since the dimension of the underlying space is 3 .

We are now in the position to state our main results concerning global weak solutions of (1.4) and nonlinear stability of the Ekman spiral.

Theorem 2.2. Assume that $u_{\infty} \delta \nu^{-1} \leq \frac{3}{2 \sqrt{2}}$. Then the following assertions hold.

a) There exists a weak solution to (1.4).

b) For every $w_{0} \in L_{\sigma}^{2}\left(\mathbb{R}_{+}^{3}\right)$ there exists at least one global weak solution $w$ of (1.4) such that

$$
\lim _{t \rightarrow \infty}\|w(t)\|_{2}=0 \text {. }
$$

c) Assume that for $w_{0} \in L_{\sigma}^{2}\left(\mathbb{R}_{+}^{3}\right)$ and some $\alpha>0$

$$
\left\|e^{-t A_{S C E}} w_{0}\right\|_{2}=O\left(t^{-\alpha}\right) .
$$

Then there exists at least one global weak solution $w$ of (1.4) such that

$$
\|w(t)\|_{2}= \begin{cases}O\left(t^{-\alpha}\right), & \alpha \leq \frac{1}{4}, \\ O\left(t^{-\frac{1}{4}}\right), & \alpha>\frac{1}{4} .\end{cases}
$$

\section{TOOLS FOR THE PROOF}

Let $1<r<\infty$ and for $f \in L^{r}\left((0, T), L_{\sigma}^{r}\left(\mathbb{R}_{+}^{3}\right)\right)$ consider the inhomogeneous equation

$$
\left\{\begin{aligned}
u^{\prime}+A u & =f, \quad t \in(0, T), \\
u(0) & =0,
\end{aligned}\right.
$$


associated with a sectorial operator $A$ in $L_{\sigma}^{r}\left(\mathbb{R}_{+}^{3}\right)$. We say that $A$ admits maximal $L^{r}$-regularity if there exists a unique function $u \in W^{1, r}\left((0, T) ; L_{\sigma}^{r}\left(\mathbb{R}_{+}^{3}\right)\right) \cap L^{r}((0, T) ; D(A))$ satisfying (3.1). In this case, there exists a constant $C>0$ such that

$$
\left\|u^{\prime}\right\|_{L^{r}\left(0, T ; L_{\sigma}^{r}\left(\mathbb{R}_{+}^{3}\right)\right)}+\|A u\|_{L^{r}\left(0, T ; L_{\sigma}^{r}\left(\mathbb{R}_{+}^{3}\right)\right)} \leq C\|f\|_{L^{r}\left(0, T ; L_{\sigma}^{r}\left(\mathbb{R}_{+}^{3}\right)\right)}
$$

holds. It is well known that the Stokes operator $A_{S}$ in $L_{\sigma}^{r}\left(\mathbb{R}_{+}^{3}\right)$ admits maximal $L^{r}$-regularity; see e.g. $[17],[6]$, or [10]. In order to prove that this holds true also for the Stokes-Coriolis-Ekman operator, we show that $A_{S C E}-A_{S}$ is relatively bounded with respect to $A_{S}$ and apply Proposition 4.3 of [5]. Observe that there exists a constant $\mu>0$ such that

$$
\mathcal{R}\left\{\lambda\left(\lambda+\mu+A_{S C E}\right)^{-1}: \lambda \in \Sigma_{\theta}\right\}<\infty,
$$

where $\Sigma_{\theta}:=\{z \in \mathbb{C} \backslash\{0\}: \arg z<\theta\}$ for some $\theta>\frac{\pi}{2}$ and $\mathcal{R}$ denotes the $\mathcal{R}$-bound of a family of bounded operators on $L_{\sigma}^{r}\left(\mathbb{R}_{+}^{3}\right)$. Thus, Proposition 4.3 in [5] implies the following proposition.

Proposition 3.1. Let $1<r<\infty, f \in L^{r}\left((0, T) ; L_{\sigma}^{r}\left(\mathbb{R}_{+}^{3}\right)\right)$ and $A_{S C E}$ in $L_{\sigma}^{r}\left(\mathbb{R}_{+}^{3}\right)$ be given as in (2.2). Then there exists $\mu>0$ such that $A_{S C E}+\mu$ admits maximal $L^{r}$-regularity on $L_{\sigma}^{r}\left(\mathbb{R}_{+}^{3}\right)$. In particular, there exists a constant $C>0$ such that

$$
\left\|u^{\prime}\right\|_{L^{r}\left(0, T ; L_{\sigma}^{r}\left(\mathbb{R}_{+}^{3}\right)\right)}+\left\|\left(A_{S C E}+\mu\right) u\right\|_{L^{r}\left(0, T ; L_{\sigma}^{r}\left(\mathbb{R}_{+}^{3}\right)\right)} \leq C\|f\|_{L^{r}\left(0, T ; L_{\sigma}^{r}\left(\mathbb{R}_{+}^{3}\right)\right)}
$$

holds.

Besides the above maximal $L^{r}$-estimates for our evolution equation, we need the following simple lemma on interpolation of $L^{p}$-spaces.

Lemma 3.2. If $1<p<q<r<\infty$, then $L^{p}\left(\mathbb{R}_{+}^{n}\right) \cap L^{r}\left(\mathbb{R}_{+}^{n}\right) \subset L^{q}\left(\mathbb{R}_{+}^{n}\right)$ and

$$
\|u\|_{q} \leq\|u\|_{p}^{\alpha}\|u\|_{r}^{1-\alpha}, \text { where } \alpha=\frac{q^{-1}-r^{-1}}{p^{-1}-r^{-1}}
$$

In fact, taking Hölder's inequality with exponents $\frac{p}{\alpha q}$ and $\frac{r}{(1-\alpha) q}$ yields the claim.

\section{The Stokes-Coriolis-Ekman Semigroup on $L_{\sigma}^{2}\left(\mathbb{R}_{+}^{3}\right)$}

In this section we show that the Stokes-Coriolis-Ekman operator generates a contraction semigroup on $L_{\sigma}^{2}\left(\mathbb{R}_{+}^{3}\right)$ provided the Reynolds number $R e=u_{\infty} \delta \nu^{-1}$ is small enough. To this end, consider the Stokes-Coriolis-Ekman operator $A_{S C E}$ in $L_{\sigma}^{2}\left(\mathbb{R}_{+}^{3}\right)$ defined by

$$
\left\{\begin{aligned}
A_{S C E} w & =-\nu P \Delta w+\Omega P J P w+\left(P\left(u_{E} \cdot \nabla\right) w+P w_{3} \partial_{3} u_{E}\right)=\left(A_{S}+A_{C}+A_{E}\right) w \\
D\left(A_{S C E}\right) & =H^{2}\left(\mathbb{R}_{+}^{3}\right) \cap H_{0}^{1}\left(\mathbb{R}_{+}^{3}\right) \cap L_{\sigma}^{2}\left(\mathbb{R}_{+}^{3}\right) .
\end{aligned}\right.
$$

Then the following result holds:

Theorem 4.1. The Stokes-Coriolis-Ekman operator $A_{S C E}$ generates an analytic $C_{0}$-semigroup $T_{S C E}$ of contractions on $L_{\sigma}^{2}\left(\mathbb{R}_{+}^{3}\right)$ provided

$$
\frac{u_{\infty} \delta}{\nu} \leq \frac{3}{2 \sqrt{2}}
$$

Note that by the results of the previous sections we already know that $T_{S C E}^{p}$ is an analytic $C_{0^{-}}$ semigroup on $L_{\sigma}^{p}\left(\mathbb{R}_{+}^{3}\right)$ for $1<p<\infty$. The uniform boundedness of $T_{S C E}$ in $L_{\sigma}^{2}\left(\mathbb{R}_{+}^{3}\right)$ will be essential in the following.

Lemma 4.2. Let $p \in[1, \infty], q \in[1, \infty)$ and $\alpha>0$. Then there exists a constant $C>0$ such that

$$
\left\|\mathrm{e}^{-(\cdot) / \alpha} w(\cdot)\right\|_{L^{p}\left(\mathbb{R}_{+}\right)} \leq C \alpha^{1-1 / q+1 / p}\left\|\frac{\mathrm{d}}{\mathrm{d} x} w\right\|_{L^{q}\left(\mathbb{R}_{+}\right)}
$$

for all $w \in W_{0}^{1, q}\left(\mathbb{R}_{+}\right)$. 
Proof. Note first that it suffices to show the assertion for $w \in C_{c}^{\infty}\left(\mathbb{R}_{+}\right)$. In this case the result then follows from the representation

$$
\mathrm{e}^{-s / \alpha} w(s)=\mathrm{e}^{-s / \alpha} \int_{0}^{s} w^{\prime}(t) \mathrm{d} t, \quad s>0,
$$

and the fact that the function $s \mapsto \mathrm{e}^{-s / \alpha}$ belongs to $L^{p}\left(\mathbb{R}_{+}\right)$for all $p \in[1, \infty]$.

Proof. of Theorem 4.1. For $w_{0} \in L_{\sigma}^{2}\left(\mathbb{R}_{+}^{3}\right)$ set $w(t):=T_{S C E}(t) w_{0}$. Then $w$ satisfies

$$
\left\{\begin{aligned}
w^{\prime}+A_{S C E} w & =0, \quad t>0 \\
w(0) & =w_{0} .
\end{aligned}\right.
$$

Multiplying the above equation with $w$ and taking into account the skew symmetry of the second and third term of $A_{S C E}$ we obtain

$$
\frac{1}{2} \frac{\mathrm{d}}{\mathrm{d} t} \int_{\mathbb{R}_{+}^{3}}|w(t)|^{2} d x+\nu \int_{\mathbb{R}_{+}^{3}}|\nabla w(t)|^{2} d x+\int_{\mathbb{R}_{+}^{3}} w(t) \cdot\left(w_{3}(t) \cdot \partial_{3} u_{E}\right) d x=0, \quad t>0 .
$$

Since

$$
\int_{\mathbb{R}_{+}^{3}} w \cdot\left(w_{3} \cdot \partial_{3} u_{E}\right) d x \leq \sum_{j=1}^{2}\left\|\mathrm{e}^{(\cdot) / 2 \delta}\left(\partial_{3} u_{E}\right)^{j} w^{3}\right\|_{2}\left\|\mathrm{e}^{-(\cdot) / 2 \delta} w^{j}\right\|_{2}
$$

and since

$$
\partial_{3} u_{E}\left(x_{3}\right)=\frac{u_{\infty}}{\delta} \mathrm{e}^{-x_{3} / \delta}\left(\begin{array}{c}
\cos \left(x_{3} / \delta\right)+\sin \left(x_{3} / \delta\right) \\
\cos \left(x_{3} / \delta\right)-\sin \left(x_{3} / \delta\right) \\
0
\end{array}\right),
$$

we see that

$$
\left\|\mathrm{e}^{(\cdot) / 2 \delta}\left(\partial_{3} u_{E}\right)^{j} w^{3}\right\|_{2} \leq \sqrt{2} \frac{u_{\infty}}{\delta}\left\|\mathrm{e}^{-(\cdot) / 2 \delta} w^{3}\right\|_{2} .
$$

The above Lemma 4.2 implies

$$
\left\|\mathrm{e}^{-(\cdot) / 2 \delta} w^{j}\right\|_{2} \leq \sqrt{\frac{2}{3}} \delta\left\|\partial_{3} w^{j}\right\|_{2}, \quad j=1,2,3 .
$$

Combining these estimates, we finally have

$$
\int_{\mathbb{R}_{+}^{3}} w \cdot\left(w_{3} \cdot \partial_{3} u_{E}\right) d x \leq \frac{2 \sqrt{2}}{3} u_{\infty} \delta\|\nabla w\|_{2}^{2} .
$$

Thus

for all $t>0$, provided

$$
\frac{\mathrm{d}}{\mathrm{d} t}\|w(t)\|_{2}^{2} \leq 0
$$

$$
u_{\infty} \delta \leq \frac{3 \nu}{2 \sqrt{2}}
$$

Therefore

$$
\left\|T_{S C E}(t) w_{0}\right\|_{2}=\|w(t)\|_{2} \leq\left\|w_{0}\right\|_{2}, \quad t>0,
$$

and the assertion is proved. 


\section{Existence OF GLOBAL WEAK SOLUTiONS}

In this section we prove the existence of a global weak solution to problem (1.4) or to problem (2.1) in the case where the Reynolds number $R e=u_{\infty} \delta \nu^{-1}$ is small enough. We therefore assume throughout this section that

$$
R e=\frac{u_{\infty} \delta}{\nu} \leq \frac{3}{2 \sqrt{2}}
$$

We subdivide the proof into three steps.

Step 1: Approximate local solutions

We start by constructing first approximate solutions to our problem. To this end, we introduce smoothing operators $J_{k}$ given by

$$
J_{k}:=\left(1+k^{-1} A_{S C E}\right)^{-1}, \quad k \in \mathbb{N} .
$$

Since $A_{S C E}$ is dissipative in $L_{\sigma}^{2}\left(\mathbb{R}_{+}^{3}\right)$, see Theorem $4.1, J_{k}$ is a bounded operator in $L_{\sigma}^{2}\left(\mathbb{R}_{+}^{3}\right)$ with $\left\|J_{k}\right\|_{L_{\sigma}^{2}\left(\mathbb{R}_{+}^{3}\right)} \leq 1$ for all $k \in \mathbb{N}$. By Sobolev's embedding theorem we have

$$
\left\|J_{k} u\right\|_{\infty} \leq C(k)\|u\|_{2} .
$$

Moreover, if $1<p<\infty$, then there exist $k_{0} \in \mathbb{N}$ and $C>0$ such that

$$
\left\|J_{k} u\right\|_{L_{\sigma}^{p}\left(\mathbb{R}_{+}^{3}\right)} \leq C\|u\|_{L_{\sigma}^{p}\left(\mathbb{R}_{+}^{3}\right)}, k \geq k_{0} .
$$

Indeed, this follows from the fact that $A_{S C E}$ generates analytic semigroup on $L_{\sigma}^{p}\left(\mathbb{R}_{+}^{3}\right)$ and general properties of sectorial operators. We now set

$$
w_{0 k}:=J_{k} w_{0} \text { and } F_{k} w:=-P\left(J_{k} w \cdot \nabla\right) w
$$

and construct approximate solutions $w_{k}$ to equation (2.1) by solving the integral equations

$$
w_{k}(t)=e^{-t A_{S C E}} w_{0 k}+\int_{0}^{t} e^{-(t-s) A_{S C E}} F_{k} w_{k}(s) d s .
$$

To this end, consider for $T>0$ the Banach space $X:=C\left([0, T] ; D\left(A_{S C E}^{1 / 2}\right)\right)$ equipped with the norm

$$
\|u\|_{T}:=\sup _{0 \leq t \leq T}\left(\|u(t)\|_{2}+\left\|A_{S C E}^{1 / 2} u(t)\right\|_{2}\right)
$$

and for $M>0$ and $k \in \mathbb{N}$ the closed set

$$
S(k, M, T):=\left\{u \in X, u(0)=w_{0 k},\|u\|_{T} \leq M\right\} .
$$

as well as the nonlinear operator $\Gamma_{k}$ defined on $S(k, M, T)$ by

$$
\Gamma_{k} u(t):=e^{-t A_{S C E}} w_{0 k}+\int_{0}^{t} e^{-(t-s) A_{S C E}} F_{k} u(s) \mathrm{d} s .
$$

Note that $\left\|F_{k} u\right\|_{2} \leq C(k)\|u\|_{2}\|\nabla u\|_{2}$. Since $D\left(A_{S C E}^{1 / 2}\right)=H_{0}^{1}\left(\mathbb{R}_{+}^{3}\right) \cap L_{\sigma}^{2}\left(\mathbb{R}_{+}^{3}\right)$ and therefore

$$
\|\nabla u\|_{2} \leq\|u\|_{2}+\left\|A_{S C E}^{1 / 2} u\right\|_{2}
$$

we see that

$$
\left\|F_{k} u\right\|_{2} \leq C(k)\left(\|u\|_{2}^{2}+\|u\|_{2}\left\|A_{S C E}^{1 / 2} u\right\|_{2}\right) .
$$

Since $e^{-t A_{S C E}}$ is an analytic semigroup of contractions we also have

$$
\left\|\nabla e^{-t A_{S C E}} w\right\|_{2} \leq C t^{-\frac{1}{2}}\|w\|_{2}, \quad t>0 .
$$


We thus may estimate $\Gamma_{k} u(t)$ as follows

$$
\begin{aligned}
\left\|\Gamma_{k} u(t)\right\|_{T} \leq & \left\|w_{0 k}\right\|_{2}+\left\|A_{S C E}^{1 / 2} w_{0 k}\right\|_{2} \\
& +\sup _{0 \leq t \leq T}\left\{\int_{0}^{t} C(k)\|u\|_{2}^{2} \mathrm{~d} s\right\}+\sup _{0 \leq t \leq T}\left\{\int_{0}^{t} C(k)\|u\|_{2}\left\|A_{S C E}^{1 / 2} u\right\|_{2} \mathrm{~d} s\right\} \\
& +\sup _{0 \leq t \leq T}\left\{\int_{0}^{t} t^{-\frac{1}{2}} C(k)\|u\|_{2}^{2} \mathrm{~d} s\right\}+\sup _{0 \leq t \leq T}\left\{\int_{0}^{t} t^{-\frac{1}{2}} C(k)\|u\|_{2}\left\|A_{S C E}^{1 / 2} u\right\|_{2} \mathrm{~d} s\right\} \\
\leq & \left\|w_{0 k}\right\|_{2}+\left\|A_{S C E}^{1 / 2} w_{0 k}\right\|_{2}+C_{1}(k) M^{2}\left(T+T^{\frac{1}{2}}\right) .
\end{aligned}
$$

Furthermore, since

$$
\begin{aligned}
\left\|F_{k} u_{1}-F_{k} u_{2}\right\|_{2} & \leq\left\|J_{k} u_{2} \nabla\left(u_{1}-u_{2}\right)\right\|_{2}+\left\|\left(J_{k} u_{1}-J_{k} u_{2}\right) \nabla u_{1}\right\|_{2} \\
& \leq C M\left\|u_{1}-u_{2}\right\|_{T}, \quad u_{1}, u_{2} \in S(k, M, T) .
\end{aligned}
$$

we obtain

$$
\left\|\Gamma_{k} u_{1}(t)-\Gamma_{k} u_{2}(t)\right\|_{T} \leq C_{2}(k) M\left(T+T^{\frac{1}{2}}\right)\left\|u_{1}(t)-u_{2}(t)\right\|_{T} .
$$

Fix now $M$ in such a way that $\left\|w_{0 k}\right\|_{2}+\left\|A_{S C E}^{1 / 2} w_{0 k}\right\|_{2} \leq \frac{M}{2}$ and then $T$ such that $C_{1}(k) M^{2}\left(T+T^{\frac{1}{2}}\right) \leq \frac{M}{2}$ and $C_{2}(k) M\left(T+T^{\frac{1}{2}}\right)<1$. Then $\Gamma_{k}$ is a strict contraction in $S(k, M, T)$ and by Banach fixed point theorem, there exists a unique $w_{k}$ in $S(k, M, T)$ satisfying $(5.3)$ for $t \in(0, T)$.

Step 2: Approximate global solutions

In the following we prove a priori bounds for $w_{k}(T)$ and $A_{S C E}^{1 / 2} w_{k}(T)$ for all $T>0$. To this end, recall that $w_{k}$ is the solution of the equation

$$
w_{k}^{\prime}(t)+A_{S C E} w_{k}=F_{k} w_{k}, \quad t \in(0, T) .
$$

Multiplying (5.6) with $w_{k}$ and integrating by parts yields

$$
\frac{1}{2} \frac{\mathrm{d}}{\mathrm{d} t}\left\|w_{k}\right\|_{2}^{2}+\left\langle A_{S C E} w_{k}, w_{k}\right\rangle=\left\langle F_{k} w_{k}, w_{k}\right\rangle
$$

Since $\left\langle\left(u_{E} \cdot \nabla\right) w_{k}, w_{k}\right\rangle=\Omega\left\langle\left(\mathbf{e}_{3} \times w_{k}\right), w_{k}\right\rangle=\left\langle F_{k} w_{k}, w_{k}\right\rangle=0$ it follows that

$$
\frac{1}{2} \frac{\mathrm{d}}{\mathrm{d} t}\left\|w_{k}\right\|_{2}^{2}+\left\langle A_{S} w_{k}, w_{k}\right\rangle+\left\langle w_{k 3} \partial_{3} u_{E}, w_{k}\right\rangle=0
$$

Theorem 4.1 implies

$$
\frac{1}{2} \frac{\mathrm{d}}{\mathrm{d} t}\left\|w_{k}\right\|_{2}^{2}+C\left\|\nabla w_{k}\right\|_{2}^{2} \leq 0
$$

for some $C>0$. Integrating with respect to $t$ yields

$$
\left\|w_{k}(T)\right\|_{2}^{2}+\int_{0}^{T}\left\|\nabla w_{k}(s)\right\|_{2}^{2} d s \leq C\left\|w_{0}\right\|_{2} .
$$

Next, forming the dual pairing of (5.6) with $A_{S C E} w_{k}$ we obtain

$$
\begin{aligned}
\left\langle w_{k}^{\prime}, A_{S} w_{k}\right\rangle+\left\langle w_{k}^{\prime},\left(u_{E} \cdot \nabla\right) w_{k}\right\rangle+\left\langle w_{k}^{\prime}, w_{k 3} \partial_{3} u_{E}\right\rangle & \\
+\left\langle w_{k}^{\prime}, \Omega\left(\mathbf{e}_{3} \times w_{k}\right)\right\rangle+\left\langle A_{S C E} w_{k}, A_{S C E} w_{k}\right\rangle & =\left\langle F_{k} w_{k}, A_{S C E} w_{k}\right\rangle .
\end{aligned}
$$

Substituting $w_{k}^{\prime}=-A_{S C E} w_{k}+F_{k} w_{k}$ leads to

$$
\begin{aligned}
\left\langle w_{k}^{\prime}, A_{S} w_{k}\right\rangle+\left\|A_{S C E} w_{k}\right\|_{2}^{2}= & \left\langle A_{S C E},\left(u_{E} \cdot \nabla\right) w_{k}+w_{k 3} \partial_{3} u_{E}+\Omega\left(\mathbf{e}_{3} \times w_{k}\right)\right\rangle \\
& -\left\langle F_{k} w_{k},\left(u_{E} \cdot \nabla\right) w_{k}+w_{k 3} \partial_{3} u_{E}+\Omega\left(\mathbf{e}_{3} \times w_{k}\right)\right\rangle+\left\langle F_{k} w_{k}, A_{S C E} w_{k}\right\rangle .
\end{aligned}
$$

Hence,

$\left\langle w_{k}^{\prime}, A_{S} w_{k}\right\rangle \leq C\left(\left\|w_{k}\right\|_{2}^{2}+\left\|\nabla w_{k}\right\|_{2}^{2}\right)+C(k)\left\|w_{k}\right\|_{2}^{2}\left\|\nabla w_{k}\right\|_{2}^{2} \leq C\left(\left\|w_{0}\right\|_{2}^{2}+\left\|\nabla w_{k}\right\|_{2}^{2}\right)+C(k)\left\|w_{0}\right\|_{2}^{2}\left\|\nabla w_{k}\right\|_{2}^{2}$, 
where the second inequality is due to the fact that $\left\|w_{k}\right\|_{2}^{2} \leq\left\|w_{k 0}\right\|_{2}^{2} \leq\left\|w_{0}\right\|_{2}^{2}$ which follows from (5.7) and the contractivity of $J_{k}$. Finally, since $\left\langle w_{k}^{\prime}, A_{S} w_{k}\right\rangle=\frac{1}{2} \frac{\mathrm{d}}{\mathrm{d} t}\left\|\nabla w_{k}\right\|_{2}^{2}$, integrating with respect to $t$ yields together with (5.8)

$$
\left\|\nabla w_{k}(T)\right\|_{2}^{2} \leq C\left(T\left\|w_{0}\right\|_{2}^{2}+\left\|w_{0}\right\|_{2}^{2}\right)+C(k)\left\|w_{0}\right\|_{2}^{4}+\left\|\nabla w_{0 k}\right\|_{2}^{2} .
$$

As $D\left(A_{S C E}^{1 / 2}\right)=H_{0}^{1}\left(\mathbb{R}_{+}^{3}\right) \cap L_{\sigma}^{2}\left(\mathbb{R}_{+}^{3}\right)$ the estimate

$$
\left\|A_{S C E}^{1 / 2} u\right\|_{2} \leq\|u\|_{2}+\|\nabla u\|_{2}
$$

holds. Combining this with the estimate given in (5.8) we obtain an a-priori bound for $\left\|w_{k}\right\|_{T}$ for every $T>0$.

Step 3: Weak convergence

In this final step we show that the approximate global solutions $w_{k}$ constructed above converge in the weak sense to some function $w$ satisfying the equation

$$
w(t)=e^{-t A_{S C E}} w_{0}-\int_{0}^{t} e^{-(t-s) A_{S C E}} P(w \cdot \nabla) w(s) d s .
$$

We fix some time interval $[0, T]$. Since

$$
\int_{0}^{T}\left\|A_{S C E}^{1 / 2} w_{k}(s)\right\|_{2}^{2} d s \leq \int_{0}^{T}\left\|w_{k}(s)\right\|_{2}^{2}+\left\|\nabla w_{k}(s)\right\|_{2}^{2} d s \leq T\|w(0)\|_{2}^{2}+\int_{0}^{T}\left\|\nabla w_{k}(s)\right\|_{2}^{2} d s
$$

the above inequality (5.8) implies

$$
w_{k} \in L^{2}\left(0, T ; D\left(A_{S C E}^{1 / 2}\right)\right) \cap L^{\infty}\left(0, T ; L_{\sigma}^{p}\left(\mathbb{R}_{+}^{3}\right)\right)=: Y=: Y_{1} \cap Y_{2}, \quad k \in \mathbb{N}
$$

and that $\left(w_{k}\right)$ is even a bounded sequence in $Y$. Since $Y_{1}$ is reflexive, there exists a subsequence of $\left(w_{k}\right)$ converging weakly in $Y_{1}$. Further, by Alaoglu's theorem, $\left(w_{k}\right)$ possesses a weak-star convergent subsequence in $Y_{2}$ and thus there exists a function $w \in Y$ with $\left(w_{k}\right)$ converging weakly to $w$ in $Y_{1}$ and $\left(w_{k}\right)$ converging in the weak-star topology to $w$ in $Y_{2}$.

Next, we write $w_{k}(t)=w_{k}^{(1)}(t)+w_{k}^{(2)}(t)$ where

$$
\begin{aligned}
w_{k}^{(1)}(t) & :=e^{-t A_{S C E}} w_{0 k}, \\
w_{k}^{(2)}(t) & :=\int_{0}^{t} e^{-(t-s) A_{S C E}} F_{k} w_{k}(s) \mathrm{d} s .
\end{aligned}
$$

Performing the same calculations which led to (5.8), we now obtain

$$
\left\|w_{k}^{(1)}(t)-w_{l}^{(1)}(t)\right\|_{2}^{2}+\int_{0}^{t}\left\|A_{S C E}^{1 / 2}\left(w_{k}^{(1)}(s)-w_{l}^{(1)}(t)\right)\right\|_{2}^{2} d s \leq C\left\|w_{0 k}-w_{0 l}\right\|_{2}^{2}, \quad k, l \in \mathbb{N} .
$$

Since $w_{0 k} \rightarrow w_{0}$ in $L_{\sigma}^{p}\left(\mathbb{R}_{+}^{3}\right)$ as $k \rightarrow \infty$ we see that $\left(w_{k}^{(1)}\right)$ and $\left(w_{k}^{(2)}\right)$ are bounded sequences in $Y$.

Next, we set $r=\frac{n+2}{n+1}$ for $n=3$. By Lemma 3.2, Hölder's and Sobolev's inequalities

$$
\left\|F_{k} w_{k}\right\|_{r} \leq C\left\|J_{k} w_{k}\right\|_{\frac{2(n+2)}{n}}\left\|\nabla w_{k}\right\|_{2} \leq\left\|w_{k}\right\|_{2}^{\frac{2}{n+2}}\left\|\nabla J_{k} w_{k}\right\|_{2}^{\frac{n}{n+2}}\left\|\nabla w_{k}\right\|_{2} .
$$

Since $\left\|\nabla J_{k} v_{k}\right\|_{2} \leq\left\|A_{S C E}^{1 / 2} v_{k}\right\|_{2}+\left\|v_{k}\right\|_{2}$ by (5.4), we see that

$$
\left\|F_{k} w_{k}\right\|_{r} \leq C\left\|w_{0}\right\|_{2}^{\frac{2}{n+2}}\left(\left\|A_{S C E}^{1 / 2} w_{k}\right\|_{2}+\left\|w_{0}\right\|_{2}\right)^{\frac{2}{r}}
$$

Further, since $\left(w_{k}\right)$ is bounded in $Y_{2}$, it follows from (5.8) that

$$
\int_{0}^{T}\left\|F_{k} w_{k}\right\|_{r}^{r} d t \leq C\left\|w_{0}\right\|_{2}^{\frac{2 r}{n+2}} \int_{0}^{T}\left(\left\|A_{S C E}^{1 / 2} w_{k}\right\|_{2}+\left\|w_{0}\right\|_{2}\right)^{2} d t \leq C(T+1)\left\|w_{0}\right\|_{2}^{\frac{2 r}{n+2}+2} .
$$

Hence, $\left(F_{k} w_{k}\right)$ is a bounded sequence in $L^{r}\left(0, T ; L_{\sigma}^{r}\left(\mathbb{R}_{+}^{3}\right)\right)$. By construction, $w_{k}^{(2)}$ is a solution of the Cauchy problem

$$
\begin{aligned}
w_{k}^{\prime}(t)+A_{S C E} w_{k}(t) & =F_{k} w_{k}(t), \quad t \geq 0, \\
w(0) & =0 .
\end{aligned}
$$


Thus our result on maximal $L^{r}$-regularity for $A_{S C E}$, i.e. Proposition 3.1, implies that $\left(w_{k}^{(2)}\right)$ is a bounded sequence in $L^{r}\left(0, T ; D\left(A_{S C E, r}\right)\right) \cap W^{1, r}\left(0, T ; L_{\sigma}^{r}\left(\mathbb{R}_{+}^{3}\right)\right)$. The operators $J_{k}$ are uniformly bounded on $L_{\sigma}^{r}\left(\mathbb{R}_{+}^{3}\right)$ in $k \geq k_{0}$; see (5.2). Thus $\left(J_{k} w_{k}^{(2)}\right)_{k \geq k_{0}}$ is a bounded sequence in $L^{r}\left(0, T ; D\left(A_{S C E, r}\right)\right.$ $\cap W^{1, r}\left(0, T ; L_{\sigma}^{r}\left(\mathbb{R}_{+}^{3}\right)\right)$ as well.

Since $\left(w_{k}\right)$ is a bounded sequence in $Y$ by $(5.8)$ and since $\left(w_{k}\right)$ and $\left(J_{k} w_{k}\right)$ are bounded in the space of maximal regularity, if follows from theorem III.2.1 in [18] and lemma 1.4.6 in [14] that $\left(w_{k}^{(2)}\right)$ and $\left(J_{k} w_{k}^{(2)}\right)_{k \geq k_{0}}$ are relatively compact in $L^{2}(K \times(0, T))$ for any fixed compact set $K \subset \mathbb{R}_{+}^{3}$. It follows that $\left(w_{k}^{(2)}\right)$ and $\left(J_{k} w_{k}^{(2)}\right)$ converge in $L^{2}\left(\mathbb{R}_{+}^{3} \times(0, T)\right)$. Therefore, $w_{k}(s) \rightarrow w(s)$ and $J_{k} w_{k}(s) \rightarrow w(s)$ for a.a. $s \in(0, T)$ for some function $w \in Y$.

Finally, we need to verify that function $w$ constructed above is in fact a weak solution of our problem (1.4). To this end, note that by the weak convergence of $\left(w_{k}\right)$ in $Y_{1}$ we have

$$
\begin{aligned}
\lim _{k \rightarrow \infty} \int_{0}^{T}-\left\langle w_{k}, \phi\right\rangle h^{\prime} \mathrm{d} t & =\int_{0}^{T}-\langle w, \phi\rangle h^{\prime} \mathrm{d} t, \\
\lim _{k \rightarrow \infty} \int_{0}^{T}\left\langle\nabla w_{k}, \nabla \phi\right\rangle h \mathrm{~d} t & =\int_{0}^{T}\langle\nabla w, \nabla \phi\rangle h \mathrm{~d} t, \\
\lim _{k \rightarrow \infty} \int_{0}^{T}\left\langle\left(u_{E} \cdot \nabla\right) w_{k}, \phi\right\rangle h \mathrm{~d} t & =\int_{0}^{T}\left\langle\left(u_{E} \cdot \nabla\right) w, \phi\right\rangle h \mathrm{~d} t, \\
\lim _{k \rightarrow \infty} \int_{0}^{T}\left\langle w_{k 3} \cdot \partial_{3} u_{E}, \phi\right\rangle h \mathrm{~d} t & =\int_{0}^{T}\left\langle w_{3} \cdot \partial_{3} u_{E}, \phi\right\rangle h \mathrm{~d} t, \\
\lim _{k \rightarrow \infty} \int_{0}^{T} \omega\left\langle\mathbf{e}_{3} \times w_{k}, \phi\right\rangle h \mathrm{~d} t & =\int_{0}^{T} \omega\left\langle\mathbf{e}_{3} \times w, \phi\right\rangle h \mathrm{~d} t, \\
\lim _{k \rightarrow \infty}\left\langle w_{0 k}, \phi\right\rangle h(0) & =\left\langle w_{0}, \phi\right\rangle h(0)
\end{aligned}
$$

since all these terms are linear. It remains to show that

$$
\lim _{k \rightarrow \infty} \int_{0}^{T}\left\langle J_{k} w_{k} \cdot \nabla w_{k}, \phi\right\rangle h \mathrm{~d} t=\int_{0}^{T}\langle w \cdot \nabla w, \phi\rangle h \mathrm{~d} t .
$$

Let $\chi_{N}: \mathbb{R}_{+}^{3} \rightarrow\{0,1\}$ be given by

$$
\chi_{N}(x)= \begin{cases}1, & x \in \mathbb{R}_{+}^{3} \cap B(0, N) \\ 0, & \text { otherwise }\end{cases}
$$

where $B(x, n)$ denotes the ball with center $x$ and radius $n$ and consider

$$
\int_{0}^{T}\left\langle J_{k} w_{k} \cdot \nabla w_{k}, \phi\right\rangle h \mathrm{~d} t=\int_{0}^{T}\left\langle J_{k} w_{k} \cdot \nabla w_{k}, \chi_{N} \phi\right\rangle h \mathrm{~d} t+\int_{0}^{T}\left\langle J_{k} w_{k} \cdot \nabla w_{k},\left(1-\chi_{N}\right) \phi\right\rangle h \mathrm{~d} t .
$$

Assume first that $w \in D\left(A_{S C E}^{1 / 2}\right) \cap L^{\infty}$. Then

$$
\lim _{N \rightarrow \infty} \int_{0}^{T}\left\langle J_{k} w_{k} \cdot \nabla w_{k}, \chi_{N} \phi\right\rangle h \mathrm{~d} t=\int_{0}^{T}\left\langle w \cdot \nabla w, \chi_{N} \phi\right\rangle h \mathrm{~d} t
$$

since $\chi_{N} v$ is bounded and has bounded support. If $v \notin L^{\infty}$, take $J_{n} w \in D\left(A_{S C E}^{1 / 2}\right) \cap L^{\infty}$ and pass then to the limit $N \rightarrow \infty$. 
Since $n=3$ we obtain by Hölder's and by Sobolev's inequality with $r=\left(\frac{1}{2}+\frac{1}{n}\right)^{-1}=\frac{6}{5}$ as well as by the Gagliardo-Nirenberg theorem that

$$
\begin{aligned}
\left|\int_{0}^{T}\left\langle J_{k} w_{k} \cdot \nabla w_{k},\left(1-\chi_{N}\right) \phi\right\rangle h \mathrm{~d} t\right| & \leq C \int_{0}^{T}\left\|J_{k} w_{k} \cdot \nabla w_{k}\right\|_{r}\left\|\left(1-\chi_{n}\right) \phi\right\|_{2^{*}} \mathrm{~d} t \\
& \leq C \int_{0}^{T}\left\|J_{k} w_{k}\right\|_{n}\left\|\nabla w_{k}\right\|_{2}\left\|\left(1-\chi_{n}\right) \phi\right\|_{2^{*}} \mathrm{~d} t \\
& \leq C \int_{0}^{T}\left\|w_{k}\right\|_{2}^{\frac{1}{2}}\left\|\nabla J_{k} w_{k}\right\|_{2}^{\frac{1}{2}}\left\|\nabla w_{k}\right\|_{2}\left\|\left(1-\chi_{N}\right) \phi\right\|_{2^{*}} \mathrm{~d} t .
\end{aligned}
$$

Since $\phi \in D\left(A_{S C E}^{1 / 2}\right)$ and $D\left(A_{S C E}^{1 / 2}\right) \hookrightarrow L^{2^{*}}\left(\mathbb{R}_{+}^{3}\right)$, we obtain

$$
\lim _{N \rightarrow \infty}\left|\int_{0}^{T}\left\langle J_{k} w_{k} \cdot \nabla w_{k},\left(1-\chi_{N}\right) \phi\right\rangle h \mathrm{~d} t\right|=0 .
$$

Since our choice of $T$ was arbitrary, there exists for every $T>0$ a weak solution $w \in L^{2}\left(0, T ; D\left(A_{S C E}^{1 / 2}\right)\right) \cap$ $L^{\infty}\left(0, T ; L_{\sigma}^{2}\left(\mathbb{R}_{+}^{3}\right)\right)$ of $(2.1)$.

\section{Proof of the stability estimates}

In this section we prove assertions a) and b) of Theorem 2.2. Note first that the solution of problem (2.1) is given by

$$
w(t)=e^{-t A_{S C E}} w_{0}-\int_{0}^{t} e^{-(t-s) A_{S C E}} P(w \cdot \nabla) w(s) d s .
$$

In order to estimate the nonlinear term, recall that

$$
\left\|\nabla e^{-t A_{S C E}} u\right\|_{2} \leq C t^{-\frac{1}{2}}\|u\|_{2}, \quad u \in L_{\sigma}^{p}\left(\mathbb{R}_{+}^{3}\right)
$$

The same estimate is of course also valid for the adjoint operator $A_{S C E}{ }^{*}$. This implies

$$
\begin{aligned}
& \left\|e^{-t A_{S C E}} P(w \cdot \nabla) w\right\|_{2}=\sup _{\|\phi\|_{2}=1}\left|\left\langle e^{-t A_{S C E}} P(w \cdot \nabla) w, \phi\right\rangle\right|=\sup _{\|\phi\|_{2}=1}\left|\left\langle w \otimes w, \nabla e^{-t A_{S C E}{ }^{*}} \phi\right\rangle\right| \\
& \leq \sup _{\|\phi\|_{2}=1}\|w \otimes w\|_{2}\left\|\nabla e^{-t A_{S C E^{*}}} \phi\right\|_{2} \\
& \leq C t^{-\frac{1}{2}}\|w\|_{4}^{2}
\end{aligned}
$$

Since $w \in D\left(A_{S C E}^{1 / 2}\right)=D\left(A_{S}^{1 / 2}\right)$, it follows that

$$
\|w\|_{4}^{2} \leq C\left\|A_{S}^{3 / 8} w\right\|_{2}^{2} \leq C\|w\|_{2}^{\frac{1}{2}}\|\nabla w\|_{2}^{\frac{3}{2}}
$$

and thus we may estimate the nonlinear term as

$$
\left\|e^{-t A_{S C E}} P(w \cdot \nabla) w\right\|_{2} \leq C t^{-\frac{1}{2}}\|w\|_{2}^{\frac{1}{2}}\|\nabla w\|_{2}^{\frac{3}{2}} .
$$

Hence,

$$
\|w(t)\|_{2} \leq\left\|e^{-t A_{S C E}} w_{0}\right\|_{2}+C \int_{0}^{t}(t-s)^{-\frac{1}{2}}\|w\|_{2}^{\frac{1}{2}}\|\nabla w\|_{2}^{\frac{3}{2}} \mathrm{~d} s
$$

By (5.7), $\frac{\mathrm{d}}{\mathrm{d} t}\|w(t)\|_{2} \leq 0$ for all $t>0$. Therefore

$$
\frac{\mathrm{d}}{\mathrm{d} t}\|w(t)\|_{2}^{2}+\frac{m}{t}\|w\|_{2} \leq \frac{m}{t}\|w\|_{2}
$$

for every integer $m>0$. Multiplying this inequality by $t^{m}$ and applying estimate (6.1), yields

$$
\frac{\mathrm{d}}{\mathrm{d} t}\left(t^{m}\|w\|_{2}\right) \leq m t^{m-1}\left\|e^{-t A_{S C E}} w_{0}\right\|_{2}+C m t^{m-1}\left(\int_{0}^{t}(t-s)^{-\frac{1}{2}}\|w\|_{2}^{2} \mathrm{~d} s\right)^{\frac{1}{4}}\left(\int_{0}^{t}(t-s)^{-\frac{1}{2}}\|\nabla w\|_{2}^{2} \mathrm{~d} s\right)^{\frac{3}{4}} .
$$


Denoting the second term on the right hand side above by $F(t)$, we obtain after integrating in $t$ and dividing by $t^{m}$

$$
\|w(t)\|_{2} \leq t^{-m} \int_{0}^{t} m \tau^{m-1}\left\|e^{-t A_{S C E}} w_{0}\right\|_{2} \mathrm{~d} \tau+t^{-m} \int_{0}^{t} F(\tau) \mathrm{d} \tau .
$$

Furthermore, we set

$$
F_{1}(t):=\int_{0}^{t}(t-s)^{-\frac{1}{2}}\|w\|_{2}^{2} \mathrm{~d} s \text { and } F_{2}(t):=\int_{0}^{t}(t-s)^{-\frac{1}{2}}\|\nabla w\|_{2}^{2} \mathrm{~d} s
$$

and estimate

$$
\begin{aligned}
t^{-m} \int_{0}^{t} F(\tau) \mathrm{d} \tau & \leq C t^{-m} \int_{0}^{t} m \tau^{m-1}\left(F_{1}(\tau)\right)^{\frac{1}{4}}\left(F_{2}(\tau)\right)^{\frac{3}{4}} \mathrm{~d} \tau \\
& \leq C m t^{-1}\left(\int_{0}^{t} F_{1}(\tau) \mathrm{d} \tau\right)^{\frac{1}{4}}\left(\int_{0}^{t} F_{2}(\tau) \mathrm{d} \tau\right)^{\frac{3}{4}} \\
& \leq C m\left(t^{-1} \int_{0}^{t} F_{1}(\tau) \mathrm{d} \tau\right)^{\frac{1}{4}}\left(t^{-1} \int_{0}^{t} F_{2}(\tau) \mathrm{d} \tau\right)^{\frac{3}{4}}
\end{aligned}
$$

Recall that $\frac{\mathrm{d}}{\mathrm{d} t}\|w(t)\|_{2}^{2}+C\|\nabla w\|_{2}^{2} \leq 0$ for all $t>0$. In particular, $\|w\|_{2} \in L^{\infty}\left(\mathbb{R}_{+}\right)$and

$$
t^{-1} \int_{0}^{t} F_{1}(\tau) \mathrm{d} \tau \leq C t^{\frac{1}{2}}
$$

and also $\|\nabla w\|_{2} \in L^{1}\left(\mathbb{R}_{+}\right)$with

$$
t^{-1} \int_{0}^{t} F_{2}(\tau) \mathrm{d} \tau \leq C t^{-\frac{1}{2}}
$$

Summing up, we proved that

$$
\|w(t)\|_{2} \leq t^{-m} \int_{0}^{t} m \tau^{m-1}\left\|e^{-t A_{S C E}} w_{0}\right\|_{2} \mathrm{~d} \tau+C t^{-\frac{1}{4}} .
$$

In order to prove assertion i), we only have to observe that $\lim _{t \rightarrow \infty}\left\|e^{-t A_{S C E}} w_{0}\right\|_{2}=0$. The assertion then follows from the estimate (6.3).

Finally, assume in addition that $\left\|e^{-t A_{S C E}} w_{0}\right\|_{2}=O\left(t^{-\alpha}\right)$ for some $\alpha>0$. The estimate (6.3) implies that $t^{-m} \int_{0}^{t} m \tau^{m-1}\left\|e^{-t A_{S C E}} w_{0}\right\|_{2} \mathrm{~d} \tau=O\left(t^{-\alpha}\right)$, too, which proves assertion ii) of our theorem.

\section{REFERENCES}

[1] A. Babin, A. Mahalov and B. Nicolaenko, 3D Navier-Stokes and Euler equations with initial data characterized by uniformly large vorticity. Indiana Univ. Math. J. 50 (2001), 1-35.

[2] W. Borchers, T. Miyakawa, On stability of exterior stationary Navier-Stokes flows. Acta Math., 174, (1995), 311382 .

[3] W. Borchers, T. Miyakawa, $L^{2}$-decay for Navier-Stokes flows in unbounded domains, with applications to exterior stationary flows. Arch. Rational Mech. Anal. 118, (1992), 273-295.

[4] C. Cao, E. Titi, Global wellposedness of the three dimensional viscous primitive equations of large scale ocean and atmosphere dynamics. Annals of Math. 165, (2007), to appear.

[5] R. Denk, M. Hieber, J. Prüss, R-Boundedness, Fourier multipliers and problems of elliptic and parabolic type, Mem. Amer. Math. Soc., 166, 2003.

[6] W. Desch, M. Hieber, J. Prüss, $L^{p}$-theory of the Stokes equation in a half-space, J. Evol. Equ.,1, (2001), 115-142.

[7] B. Desjardins, E. Grenier, Linear instability implies nonlinear instability for variuos types of viscous boundary layers, Ann. I.H. Poincaré, 20, (2003), 87-106.

[8] V.W. Ekman, On th influence of the earth's rotation on ocean currents. Arkiv Matem. Astr. Fysik, (Stockholm) 11, (1905), 1-52.

[9] G.P. Galdi, An Introduction to the Mathematical Theory of the Navier-Stokes Equations I, Springer, 1998.

[10] M. Geissert, M. Hess, M. Hieber, C. Schwarz, K. Stavrakidis, Maximal $L^{p}-L^{q}$-Estimates for the Stokes Equation: a Short Proof of Solonnikov's Theorem, J. Math. Fluid Mech., to appear.

[11] Y. Giga, K. Inui, A. Mahalov and S. Matsui, Navier-Stokes equations in a rotating frame in $\mathbb{R}^{3}$ with initial data nondecreasing at infinity, Hokkaido Math. J. 35, (2006), 321-364.

[12] Y. Giga, K. Inui, A. Mahalov, S. Matsui and J. Saal, Rotating Navier-Stokes equations in $\mathbb{R}_{+}^{3}$ with initial data nondecreasing at infinity: the Ekman boundary layer problem, Arch. Rational Mech. Anal., to appear.

[13] H.P. Greenspan, (1968), The Theory of Rotating Fluids, Cambridge University Press. 
[14] V.G. Maz’ja, (1985), Sobolev Spaces, Springer-Verlag.

[15] T. Miyakawa, H. Sohr, Weak solutions of Navier-Stokes equations, Math. Z. 199, (1988), 455-478.

[16] H. Sohr. The Navier-Stokes Equations. An Elementary Functional Analytic Approach., Birkhäuser, Basel, 2001.

[17] V. A. Solonnikov. Estimates for solutions of nonstationary Navier-Stokes equations. J. Soviet Math. 8, (1977), 213-317.

[18] H. Temam, (1992), Navier Stokes equations, Monographs in Mathematics, 78, Basel-Boston-Stuttgart: Birkhäuser Verlag.

Technische Universität Darmstadt, Fachbereich Mathematik, Schlossgartenstr. 7, D-64289 Darmstadt, GERMANY

E-mail address: hess@mathematik.tu-darmstadt.de

Technische Universität Darmstadt, Fachbereich Mathematik, Schlossgartenstr. 7, D-64289 Darmstadt, Germany

E-mail address: hieber@mathematik.tu-darmstadt.de

Department of Mathematics, Arizona State University, Tempe, AZ 85287-1804, USA

E-mail address: mahalov@asu.edu

Universität Konstanz, Fachbereich Mathematik und Statistik, Box D 187 D-78457 Konstanz, Germany

E-mail address: juergen.saal@mathematik.uni-konstanz.de 